\title{
Cloud-aided SDR Solution for Lane-Specific Vehicle Positioning via Local Interference Compensation
}

\author{
Brian Niehoefer \\ Dortmund TU University \\ Dortmund, Germany \\ brian.niehoefer@tu-dortmund.de \\ Sarah Lehnhausen \\ Dortmund TU University \\ Dortmund, Germany \\ sarah.lehnhausen@tu-dortmund.de
}

\author{
Florian Schweikowski \\ Dortmund TU University \\ Dortmund, Germany \\ florian.schweikowski@tu-dortmund.de \\ Christian Wietfeld \\ Dortmund TU University \\ Dortmund, Germany \\ christian.wietfeld@tu-dortmund.de
}

\begin{abstract}
The steadily increasing traffic density is causing enormous negative effects such as jams, accidents or $\mathrm{CO}^{2}$ emissions, especially in urban areas. In Europe more than $12 \%$ of the traffic network is daily congested. Hereby a reliable and dynamic congestion forecast is the key to avoid and/or compensate such locale bottleneck situations. Hence, this contribution focuses on a cloud-aided, lane-specific position determination of vehicles using a so-called Local Interference Compensation to enable a more detailed and lane-accurate traffic prediction (e.g. detecting short-dated roadworks or car breakdowns), and by that trafficflow manipulation in the future. Thereby, the scientific challenge is to predict, quantify and compensate the inevitable local impacts to the positioning accuracy when using ordinary GNSS receivers. Beneath the detailed explanation of the LOCATe system itself, this contribution will also provide quantitative experimental validation and performance evaluation tests using an Advanced Software-Defined GNSS Receiver solution on georeferenced points, which fit the definition of the introduced urban canyons.
\end{abstract}

\section{TABle of Contents}

1 INTRODUCTION.............................

2 SOURCES OF Possible NON-CONFORMANCES WITHIN GNSS ........................... 2

3 Reactive ERror Compensation TeCHNIQUES WITHIN GNSS ....................

4 LOCATE - LOCAL INTERFERENCE COMPENSATION FOR GNSS ........................ 3

5 VAlidation Equipment: AdVANCED SoftWARE DEFINED GNSS RECEIVER (ASDR) ..... 4

6 ACCURACY ENHANCEMENT USING LOCATE ..

7 Conclusion ...............................

ACKNOWLEDGMENTS .........................

REFERENCES .................................

BIOGRAPHY

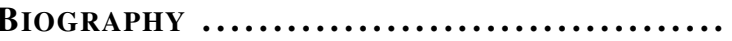

\section{INTRODUCTION}

Capability and benefit of existing or future applications are directly correlated with their reliability. Especially when talking about location-based services, the accuracy of the corresponding positioning method is a key performance indicator. This fact is even more important within traffic-relevant and by that safety-critical systems. A well-known use-case for improved positioning accuracy is the nowadays traffic situa- tion. It is a known problem without a comprehensive solution: $12 \%$ of European traffic network is permanently affected by congestion, that comes along with delays, unnecessary energy consumption and additional accidents. Hereby, a reliable and dynamic traffic forecasts is the key to avoid jams in their early beginning. However, today's traffic predictions are mainly based on unreliable and dedicated measurement points that effects the forecast results accordingly. To enable and further improve such predictions, the Communication Networks Institute (CNI) focuses, beside other tasks, on a lane-specific positioning of vehicles to enable a more detailed and lane-accurate traffic prediction in the future using multiple vehicles as combined anonymous sensor group within the traffic flow itself. Hereby, the positioning accuracy is a major key, to increase the detection rate of any traffic-affecting event. Thereby the usage of satellite-based localization techniques is obvious, since the corresponding receivers are nowadays standard in nearly every vehicle. But local effects are decreasing their performance, especially in challenging environments like urban canyons, and by that areas with a high probability of congested traffic situation. Former publications already clarified the high influences of local effects and the necessity of compensating them to provide position-continuous and highly accurate applications [1][2]. Additionally, further scenarios are conceivable which might take advantage of such improvements, like navigation for the blind, augmented reality or even Micro-UAVs like in [3].

In contrast to other scientific approaches, this contribution does not focus on the avoidance of accruing failures. Instead the so-called Local Interference Compensation (LOCATe) tries to predict, quantify and compensate the inevitable local impacts to the positioning accuracy when using ordinary GPS/GNSS receivers. Using LOCATe, commercially available and resource-constrained one-frequency receivers outperform in terms of accuracy and integrity, what will be shown using an Advanced SDR GNSS implementation. In addition, the compensation of atmospheric effects using different existing models will be evaluated and compared. To sum it up, this contribution provides a short description of existing error sources for GNSS in Section 2 and an enumeration of existing and potential future countermeasures in Section 3. Concept, description and prototypical realization of so-called LOcal interference CompensATion (LOCATe) are discussed in Section 4, followed by a brief insight to the used validation setup in Section 5 and the gained results in Section 6. 


\section{Sources of Possible NON-CONFORMANCES WITHIN GNSS}

Despite all progress in satellite and localization technology, there are still many elements of uncertainty in evaluating the satellite positioning accuracy for a specific point on earth to a given time. Figure 1 clarifies the main influences.

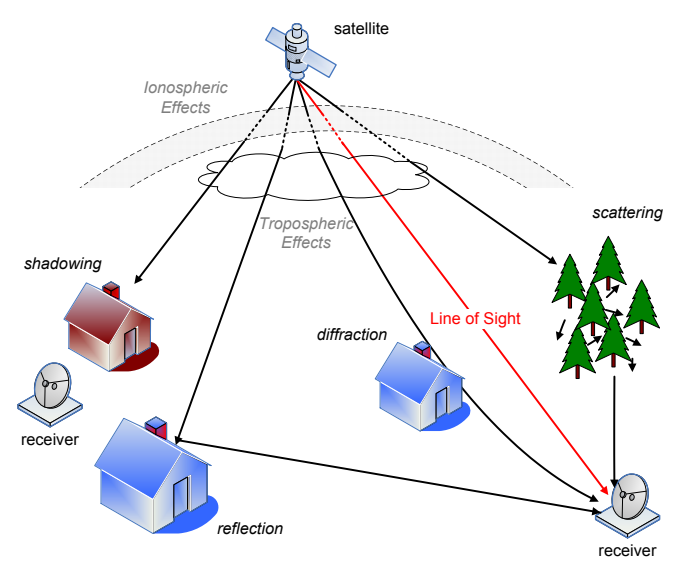

Figure 1. Elements of Uncertainty within Satellite Links

Satellite signals are subject to different atmospheric effects, like tropospheric attenuation or group delays through ionospheric refraction. The direct receiver's surrounding is another example, which highly affects the positioning precision. While a direct Line-of-Sight to four or more GPS satellites mostly enables an adequate positioning, more challenging surroundings, like an inner city or mountainous areas, leads to a significantly decreased accuracy. Especially in areas with a high probability of multipath effects, increased deviations within GNSS positioning are visible and have to be compensated to enable reliable and lane-specific applications. Within the upcoming paragraphs, a short insight in those influences will be given to enable a better understanding for the overall compensation approach of LOCATe.

\section{Atmospheric Impairments}

On its way from the GNSS satellite to a receiver the signal passes different layers of the atmosphere. Due to the direct influence to the transmission time, the ionosphere, and to a small extent the troposphere, is of primarily importance for satellite navigation signals [4]. As explained in [5], the ionosphere is caused by solar radiation, separates electrons and ions from each other. Thereby the intensity of the solar activity also affects the degree of ionization, depending on the time of day, season and location. Because of the different ionization level the density of the ionosphere and the underlaying neutrosphere and the overlying plasmasphere also differs substantially. Hence, a signal crossing those densitytransitions is affected by refraction effects and thereby the transmission time, which is necessary for the position estimation within GNSS, is also distorted in comparison to the real satellite/receiver distance [4]. This is usually in a range between 5 and $10 \mathrm{~m}$. Under extreme but also rarely accruing circumstances like solar storms, the error may reach something around a few hundred meters.

The tropospheric effects are the second atmospheric component and results from several heterogeneous weather phenomena and by that, are hard to predict. The occurring refraction depends on the height of the receiver, the elevation, the temperature, pressure and humidity next to the receiver [6].
The tropospheric refraction combines all occurring in the neutrosphere refraction effects together. Around $90 \%$ of them are caused by the dry portion of the atmosphere and can be very accurately determined. The remaining $10 \%$ are highly dependent on the locally prevailing weather situation and are only very short-term predictable. [7] describes how the various constraints affect the error. Thereby, the elevation has the highest impact. At elevation angles close to $90^{\circ}$, the satellite is directly above the user, and by that the path through the troposphere is short. Thus, the errors of about $2 \mathrm{~m}$ remains relatively small. At a low elevation, the fault may reach $25 \mathrm{~m}$ and by that it may render the use of GNSS for lane-specific applications impossible.

\section{Multipath - Position-Specific Influences}

Especially in an urban area, multipath effects are a wellknown and hot research topic in satellite navigation, the socalled urban canyons. Thereby, the signal may reach the receiver by a different route than direct one. Multipath effects like shadowing, reflection or scattering which always affect navigation signals and are the remaining error sources most difficult to predict [8][9][10]. In addition, multipath components are directly correlated with the local circumstances and by that they vary greatly and are hard to quantify [11]. Because all of them directly influence the transmission time, they also affect the Time-of-Arrival (TOA) positioning estimations of the receiver, which is used to calculate the pseudorange between a satellite and a navigation satellite receiver. Pseudo in this case is used as a term for potential incorrect positioning based on the mentioned error sources. As the measured transmission time for the GNSS signals is used, there will be errors in the calculation. This can range from a few nanoseconds in the meter range already in a roundabout way and should be compensated to enable generally valid and lane-specific usage of GNSS.

\section{REACTIVE ERROR COMPENSATION TECHNIQUES WITHIN GNSS}

Most existing solutions and approaches to reduce complex effects on satellite navigation signals may be summarized as a kind of Pre-Processing approaches and will just be enumerated in the following, because LOCATe is based on another idea. Antenna arrays, for example, would be an obvious possibility for compensating multipath effects, but require additional hardware and would increase the costs of any upcoming system [12]. The same holds true for Pseudolites Navigation [13], Multisensor Integrated Navigation [14], Antenna Design Techniques [15] or Autoregressive Modeling of INS Sensor Errors [16].

Post-Processing compensation approaches like LOCATe mainly accept unavoidable residual errors and try to calculate and compensate them afterwards. Differential GPS (D-GPS) for example, may reduce the accruing error with the use of stationary reference stations, but the benefit is directly correlated to the equality of the local circumstances between the reference station and the receiver and hence not generally valid. Other scientific approaches like [17] use an elimination of Non-Line-of-Sight (NLOS) signals, leading to a better positioning performance but also to a significant reduction of the used number of satellites in challenging areas and thus to a decreased integrity. Hence, a reception of more than four satellites is urgently required but cannot be ensured, especially in urban areas. In further studies [11], the so-called Intelligent Urban Positioning uses various approaches, such as a continuity test and a detection with the NLOS Shadow 
matching [18] combined to enable more accurate positioning. Thereby, the received signal indicators are used to improve a formerly roughly estimated position. A related idea is focused by the Shadow Matching principle. Based on an estimated position and a 3D model of the direct receiver's surrounding, a comparison between the received and their Line-of-Sight probability is made. If a signal can only be received as NLOS, it is lower weighted or even neglected in the positioning routine. The disadvantage of both techniques is the first position estimation. A bad one may end up in a deterioration using this procedure.

In contrast, our idea of LOcal interference CompensATion (LOCATe) focuses on a compensation of position-specific and unavoidable failures within GNSS positioning techniques without any additional hardware in the embedded resourceconstrained front-end. This service might be understood as a kind of simulation-based D-GPS with position-continuous virtual reference stations. Based on a highly detailed 3D model of the direct receiver surroundings including material properties, the LOCATe system simulates highly accurate satellite movement of any actual or future GNSS constellation and by that also their signals, and evaluates the impulse response using ray tracing technology. Taking all influences into account, atmospheric as well as multipath effects, a Time-of-Arrival routine is used to calculate the real receiver's position and by that the differences to the supposed one by the GNSS. The difference in between is transferred to ordinary resource-constrained one-frequency GNSS receivers afterwards.

\section{LOCATE - LOCAL INTERFERENCE COMPENSATION FOR GNSS}

As mentioned in Section 3, most GNSS improvement techniques focus on the prevention of positioning failures. In contrast, LOCATe allows a certain kind of residual error and faces their compensation afterwards. This section will give a short overview about the idea itself as well as a detailed description of the simulation architecture and further potential enhancements.

\section{Main idea of LOCATe - Predict, Quantify, Compensate}

Section 2 already discussed the different error sources to satellite navigation signals. The idea of LOCATe is to predict all this partially position-specific and highly variable influences for any given location. Thereby, LOCATe performs the following three steps:

\section{Predict all influences}

2. Quantify the accruing shift

3. Compensate the overall error vector

The procedure itself is visualized in Figure 2. First of all, the defective GNSS position $P_{G P S}$ (in this case the authors used GPS) is measured. Afterwards a reference grid around $P_{G P S}$ (blue crosses) is generated within a 3D model for the local considerations (see Section 4), whereby its dimensions are correlated to the estimated accuracy of the measured position using the available key performance indicators, like the Geometric Dilution of Precision (GDOP). Afterwards LOCATe simulates the accruing error effects (blue arrows), atmospheric as well as multipath, for every single reference point/satellite connection using the Multiscale Simulation Environment (MSE) [19]. In a further step, the most probable error vector, which ends up in the measured GPS position is used as best-in-case compensation for the actual constel-

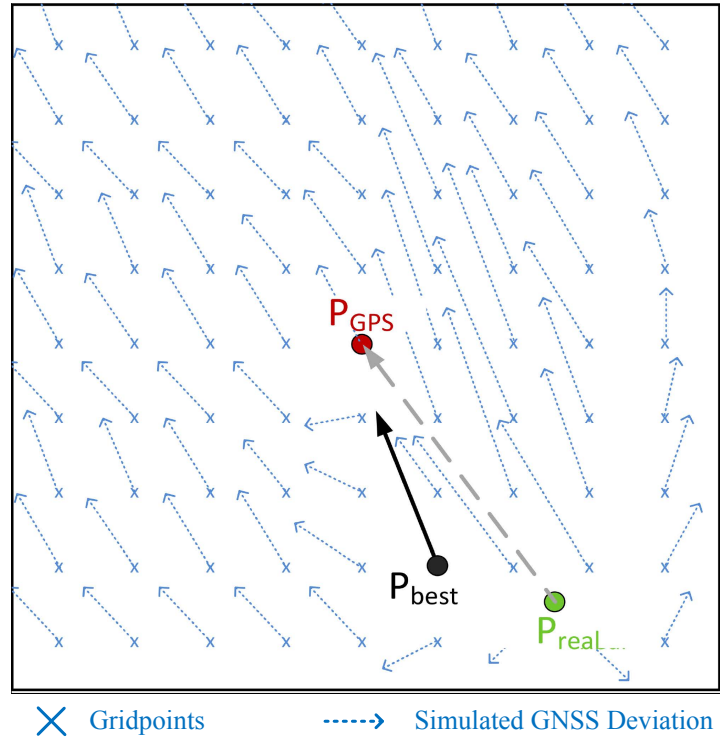

Figure 2. Evaluation of LOCATe's Enhanced GNSS Positioning

lation. The beginning of this vector then is interpreted as the supposed position $P_{\text {best }}$. To evaluate the performance of LOCATe, the authors use two of measurement points specified by the land surveying office at the campus of the university in Dortmund with fixed GPS coordinates (cmp. Section 6). This points are used for all the measurements and therefore the real position $\left(P_{\text {real }}\right)$ is known and the remaining positioning error may be quantified.

In the following, the architecture of LOCATe as well as the compensation techniques of the different error sources will be addressed.

\section{Architecture of LOCATe}

The architecture of the LOCATe is shown in Figure 3. Input

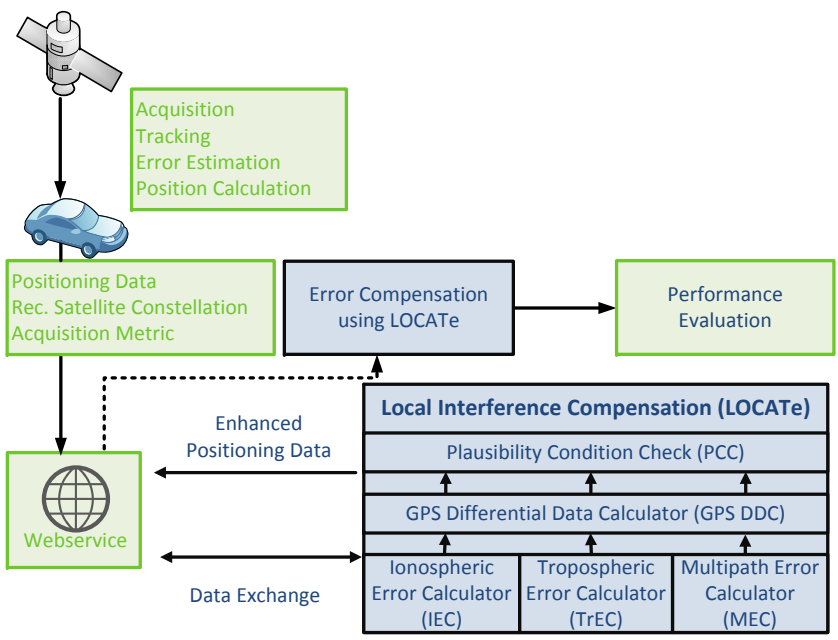

Figure 3. Architecture of LOCATe

value is the defective GPS position that is passed to the so developed Open Source Satellite Simulator $\left(O S^{3}\right)$ [20], which is based on updated Two-Line-Element sets, to enable and integrate a highly accurate satellite movement. The satellite and receiver positions are passed through multiple error 
correction modules. The atmospheric compensation modules uses SISNeT to receive EGNOS messages as additional information. Subsequently, the individual error values are determined. Using the so-called Differential Data Calculator $(D D C)$ the grid point with the highest probability of being the wanted real position is selected. Finally a validation (Plausibility Check Condition (PCC)) is performed, which determines whether time and/or position of sequenced measurements differ widely and are therefore might be subject to errors.

\section{Atmospheric Considerations}

As described above, several atmospheric effects are influencing satellite signals, and by that have to be compensated. The two main ones are ionosphere and troposphere, whose compensation methods within LOCATe will be both explained in the following.

Ionospheric Compensation-The calculation of ionospheric refraction is performed by the so-called Ionospheric Error Calculator (IEC) (cmp. Figure 3). Therefore a single-layer model is used to compress the whole effect on a certain altitude. Using the known position of the satellites using the developed $\mathrm{OS}^{3}$ and the roughly estimated one from the receiver, it becomes possible to calculate the intersection point of the signal through the ionosphere. In parallel, information from Satellite Based Augmentation Systems (SBAS) are integrated. In this case, the authors use the corresponding EGNOS messages via SISNeT [21] to gain actual values for the Ionospheric Grid Points (IGPs). Afterwards, the nearest IGPs are extracted and their values are interpolated to reach a realistic statement for the calculated Ionospheric Pearce Point (IPP) using the Total Vertical Electron Content (TVEC) using Formula 1 [4].

$$
\triangle s_{\text {iono }, p}=-F_{p p} \cdot \frac{40,3\left[\mathrm{~Hz}^{2} \mathrm{~m}^{3}\right]}{f^{2}} \cdot T V E C
$$

Hereby, $f$ stands for the used frequency and $F_{p p}$ for the obliquity factor, which is shown in Formula 2 in detail.

$$
F_{p p}=\left[1-\left(\frac{R_{0} \cdot \cos (\epsilon)}{R_{0}+h_{m}}\right)^{2}\right]^{-\frac{1}{2}}
$$

with $h_{m}$ as the altitude of the ionosphere single layer model and $R_{0}$ for the average radius of the Earth and $\epsilon$ for the specifically given elevation angle. As a last step, the corresponding error for the given satellite/earth-receiver constellation is forwarded to the Differential Data Calculator $(D D C)$, to generate the overall error vector for the actual constellation.

Tropospheric Compensation-In contrast to the ionosphere, the troposphere behaves for frequencies up to about $15 \mathrm{GHz}$ as a non-dispersive medium [22], thus no time differences between group and phase velocity. Therefore, a calculation of the error of the two-frequency method is excluded, but may be modeled using the so-called UNB3 model [23]. Based on the very variable character of the troposphere, there is no possibility to simulate or calculate it for any position on earth properly. Hence, the authors implemented the model within LOCATe's Tropospheric Error Calculator (TrEC), shown in Figure 3, as the only statistical model within the tool-chain.
Just as a rule of thumb: The UNB3 error distribution mainly follows a negative exponential behavior with increasing elevation angle, that makes sense concerning to the shorter path through the troposphere. In general those failures are limited to a few meters and can be reduced even more using the UNB3 [23].

\section{Multipath Compensation}

The Multipath Error Calculator (MEC) in Figure 3 was already explained in detail in [2] and therefore will be just mentioned shortly in this contribution. Based on a detailed 3D model of the direct receiver surroundings, the MEC simulates accurate satellite movements of any actual or future GNSS constellation using $\mathrm{OS}^{3}$. At the moment, this database is limited to the university campus $\left(51.4925^{\circ} \mathrm{N}\right.$ and $\left.7.4128^{\circ} \mathrm{E}\right)$, but therefore includes all necessary parameters to set up a highly realistic ray tracing scenario, like material properties or geo-referenced models of all buildings. The idea is to

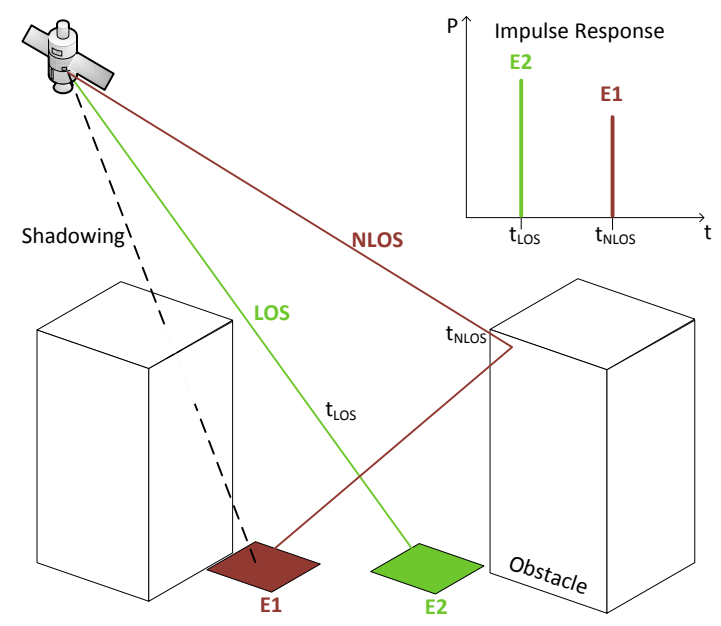

Figure 4. Using impulse response to determine ToA values

use the impulse response of the strongest received signal multipath component for the positioning as long as it is still detectable $(>-189 \mathrm{dBm})$, to determine the corresponding transmission time, like shown in Figure 4. The additional delay, compared to the transmission time in LOS, is then added to the ToA positioning routine in the DDC.

\section{VAlidation EQUiPMENT: ADVANCED SOFTWARE DEFINED GNSS RECEIVER (ASDR)}

Next to the development and the simulative validation of LOCATe itself, the authors also focused on an experimental evaluation of its performance. Using a GPS measurement equipment in combination with two GPS reference points at the campus of the university, an adequate and quantitative statement about the performance of LOCATe becomes possible. But commercially available GNSS receivers also come along with the disadvantage that there is or might be some filtering routines improving the measured positions. Hence the authors decided to use an GNSS receiver developed on their own, shown in Figure 6, based on a Software-Defined Radio $(S D R)$. This method allows the tracking of GNSS (in our case GPS) raw data. Next to the basic functionalities of GNSS receiver implementations for SDRs [24], a former publication also addresses some modifications and improvements respectively, which are visualized in Figure 5 [25] and can be 
compared to the corresponding parts of Figure 3.

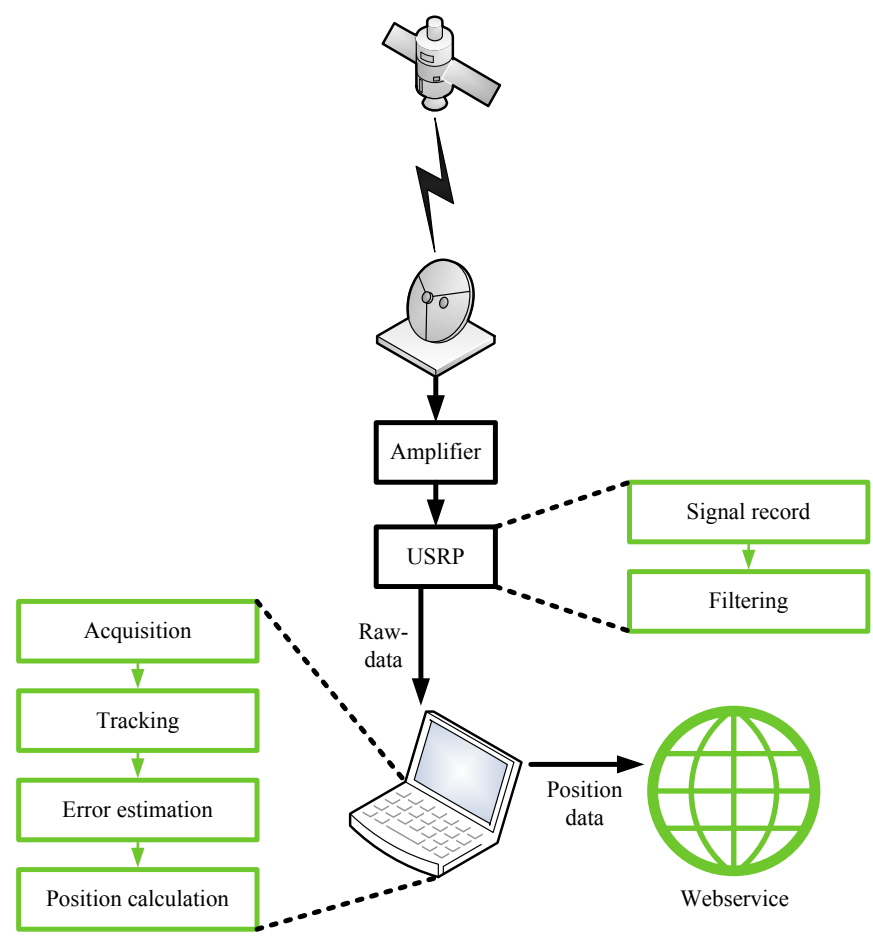

Figure 5. Functionalities of the Advanced Software-Defined GNSS Receiver

The modifications mainly deal with the overall performance and the possibility to communicate with a back-end. Therefore, the filtering routine was integrated within the ASDR implementation. This comes along with the advantage to monitor the filtering steps during runtime in the field and that allows a better parallelization. A further modification is facing a dual-link architecture to allow back-end applications to increase the positioning performance via post-processing and send those back to the receiver, to fit the idea of a cloud aided SDR solution. Using the ASDR it becomes possible to measure raw GPS or Glonass positioning data, transmit it to the LOCATe system and improve the accuracy there. To analyze the corresponding performance gain like shown in Figure 3 and explained in Section 6, reference points are necessary. Two of those were determined by the land

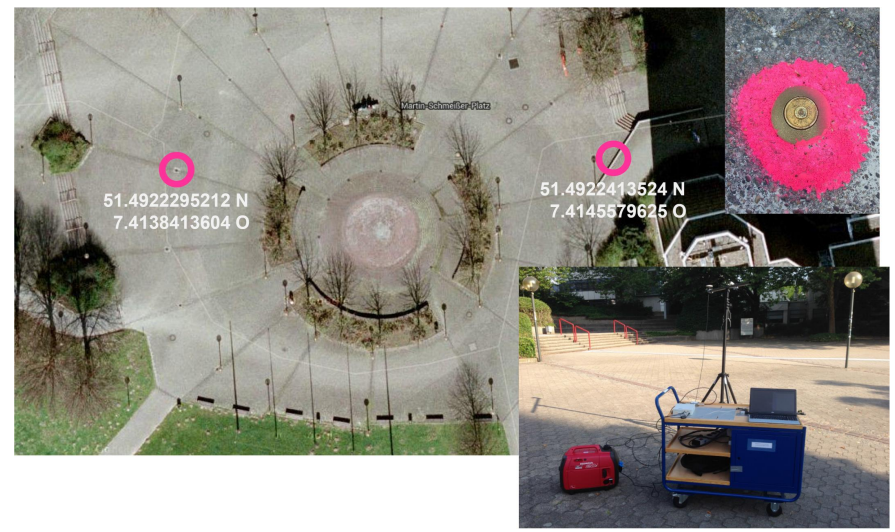

Figure 6. GPS Reference Points at the Campus and the ASDR Implementation (lower right)

surveillance office of Dortmund, with an accuracy of below
$2 \mathrm{~cm}$ and are shown in Figure 6. Both points are matching to the definition of the introduced urban canyons and by that, standing for the most challenging environments for satellite positioning systems.

\section{ACCURACY ENHANCEMENT USING LOCATE}

Section 5 already discussed the idea of using geo-referenced GPS points and the ASDR to evaluate the performance of LOCATe. The results of analyzing different LOCATe compensation setups using more than 500 measurements on the mentioned points are shown in Figure 7. It turns out that the best performance gain occurs when using the GPS integrated troposphere correction, whereby ionosphere and multipath effects are compensated by the LOCATe. Pure

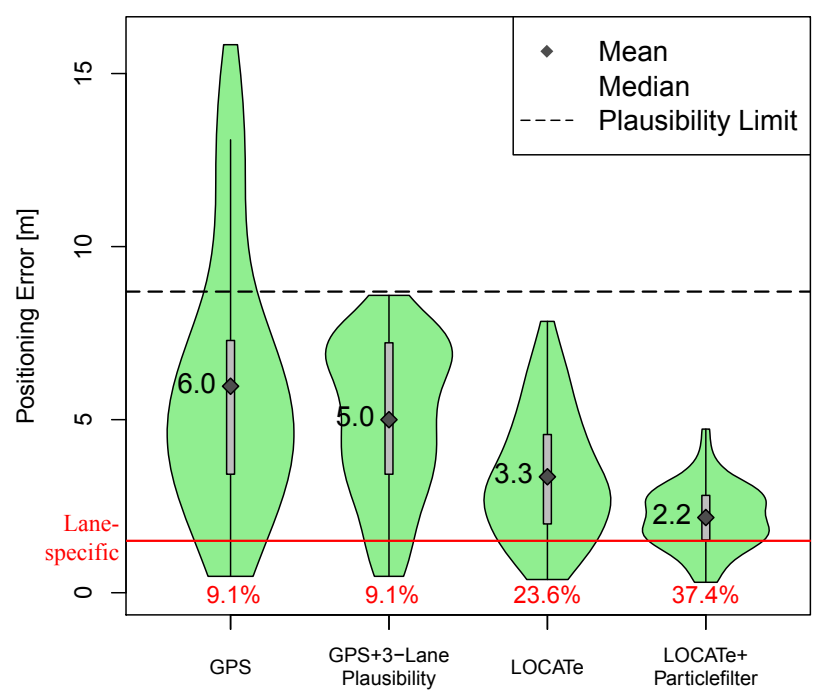

Figure 7. Performance Evaluation of LOCATe (3-Lane Plausibility)

GPS for example shows an expectable deviation for highly challenging environments, which also fits to former evaluations [2]. Based on the fact that the scenario deals with traffic situations, it is obvious to add plausibility routines, like normal GPS receiver do. E.g. on a three lane highway, deviations greater than the overall street width (e.g. a three lane highway with $2.9 \mathrm{~m}$ each lane) may be detected easily. Hence, a corresponding filter was added to the pure GPS and set all values above the plausibility limit to the nearest lanecenter value, so in this case to $7.25 \mathrm{~m}\left(=3 \cdot 2.9 \mathrm{~m}-\left(\frac{2.9 \mathrm{~m}}{2}\right)\right)$. Based on that, LOCATe used these values as input and it turns out that up to $23.6 \%$ of all positions can be determined lanespecific $(<1.5 \mathrm{~m}$, visualized by the red solid line $)$ in contrast to just $9 \%$ with pure GPS and reduce the average error by more than $45 \%$, and even more important: LOCATe lowers the occurring peak value significantly that again clarifies the benefit.

Additionally, filter algorithms may be used to further improve the accuracy gain. It should be mentioned that this point is just used to clarify the additional performance possibility and shall not indicate the finest choice to work with LOCATe. Just as an example, the authors applied a particle filter (wellknown method in GPS positioning techniques) to the already smoothed results from LOCATe and by that decreases the mean error by further $33 \%$, resulting in an overall reduction 
of $63.3 \%$ in average. Furthermore, a combined handling using LOCATe and additional filters, increases the lanespecific detection by four times. Using our measurements, more than $37 \%$ of all recorded points might be improved to a lane-specific positioning. In contrast to just $9 \%$ using GPS only. The same observations holds true for changing the mentioned plausibility limit to a two-lane highway, shown in Figure 8. Again, all values above the plausibility limit are

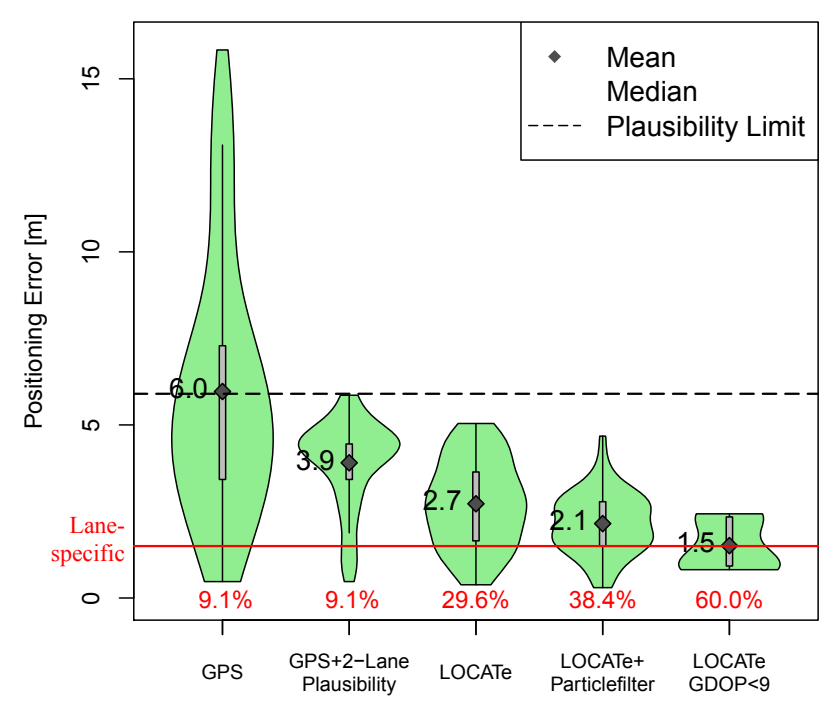

Figure 8. Performance of LOCATe with 2-Lane Plausibility and an Exemplary Scenario with Good Inputs (GDOP < 9)

mapped to the nearest lane-center value. In this case to $4.35 \mathrm{~m}$ $\left(=2 \cdot 2.9 \mathrm{~m}-\left(\frac{2.9 \mathrm{~m}}{2}\right)\right)$. LOCATe as well as the additional particle filter again performs very well with the better input values. Furthermore the effect of less distorted input values is shown the fifth example in Figure 8. Hereby, only those GPS values with a GDOP lower 9, were forwarded to LOCATe to clarify its performance in less challenging environments. Again, LOCATe is able to increase the positioning accuracy to $1.5 \mathrm{~m}$ in average and locate $60 \%$ of all values lane-specific.

\section{Conclusion}

This contribution provides the conceptional design, architecture and performance evaluation of the so-called Local Interference Compensation (LOCATe) for Global Navigation Satellite Systems, to increase the accuracy especially for lane-specific applications in urban areas. Using this postprocessing, and by that cloud-aided approach, the authors have identified certain accuracy enhancements using the three steps: Predict, quantify and compensate all influences to satellite signals at a specific point on earth. To enable a detailed and accurate performance evaluation, an Advanced Software-Defined GNSS Receiver in combination with two GPS reference points in a highly challenging urban area were used. It turns out, that using LOCATe on its own, an enhancement of up to $45 \%$ in average is possible. By adding additional filter methods to the already smoothed results from LOCATe, an overall reduction of $63 \%$ in average is visible. In addition, LOCATe eliminated the occurring peak values in GNSS and by that, allows the use of satellite-based positioning for further applications than today, even safetycritical ones.

\section{ACKNOWLEDGMENTS}

The work on this paper has been partially founded by Deutsche Forschungsgemeinschaft (DFG) within the Collaborative Research Center SFB 876 "Providing Information by Resource-Constrained Analysis", project B4 and by the European Union Seventh Framework Programme (FP7/20072013) under grant agreement 261769.

\section{REFERENCES}

[1] B. Niehoefer, A. Lewandowski, and C. Wietfeld, "Evaluation of the Localization Accuracy of Satellite Systems for Traffic Flow Predictions," in 24th International Technical Meeting of The Satellite Division of the Institute of Navigation (ION GNSS 2011), Portland, Sep 2011.

[2] B. Niehoefer, S. Lehnhausen, and C. Wietfeld, "Combined Analysis of Local Ionospheric and Multipath Effects for Lane-Specific Positioning of Vehicles within Traffic Streams," in 6th ESA Workshop on Satellite Navigation Technologies (NaviTech), Noordwijk, The Netherlands, December 2012.

[3] N. Goddemeier, K. Daniel, and C. Wietfeld, "RoleBased Connectivity Management with Realistic Airto-Ground Channels for Cooperative UAVs," Selected Areas in Communications, IEEE Journal on, vol. 30, no. 5, pp. 951-963, 2012.

[4] E. D. Kaplan and C. J. Hegarty, Understanding GPS: principles and applications. Artech house, 2005.

[5] B. Arbesser-Rastburg, "Ionospheric and Tropospheric Modelling and Monitoring for GNSS at the European Space Agency," in General Assembly and Scientific Symposium, 2011 XXXth URSI. IEEE, 2011, pp. 14.

[6] T. A. Skidmore and F. van Graas, "An Investigation of Tropospheric Errors on Differential GNSS Accuracy and Integrity," in Proceedings of the 17th International Technical Meeting of the Satellite Division of The Institute of Navigation (ION GNSS 2004), 2001, pp. 27522760.

[7] E. Altshuler, "Tropospheric Range-Error Corrections for the Global Positioning System," Antennas and Propagation, IEEE Transactions on, vol. 46, no. 5, pp. 643649, 1998.

[8] T. Sarkar, Z. Ji, K. Kim, A. Medouri, and M. SalazarPalma, "A Survey of various Propagation Models for Mobile Communication," Antennas and Propagation Magazine, IEEE, vol. 45, no. 3, pp. 51 - 82, june 2003.

[9] H.-W. Son and N.-H. Myung, "A Deterministic Ray Tube Method for Microcellular Wave Propagation Prediction Model," Antennas and Propagation, IEEE Transactions on, vol. 47, no. 8, pp. $1344-1350$, aug 1999.

[10] G. Athanasiadou, A. Nix, and J. McGeehan, "A Microcellular Ray-tracing Propagation Model and Evaluation of its Narrow-band and Wide-band Predictions," Selected Areas in Communications, IEEE Journal on, vol. 18, no. 3, pp. $322-335$, march 2000.

[11] P. D. Groves, Z. Jiang, L. Wang, and M. K. Ziebart, "Intelligent Urban Positioning using Multi-Constellation GNSS with 3D Mapping and NLOS Signal Detection," Proc. ION GNSS, 2012. 
[12] C. E. McDowell, "GPS multipath mitigation using a multi-element antenna array," Jul. 16 2002, US Patent $6,421,000$.

[13] H. So, T. Lee, S. Jeon, C. Kim, C. Kee, T. Kim, and S. Lee, "Implementation of a Vector-based Tracking Loop Receiver in a Pseudolite Navigation System," Sensors, vol. 10, no. 7, pp. 6324-6346, 2010.

[14] S. Gao, Y. Zhong, X. Zhang, and B. Shirinzadeh, "Multi-sensor Optimal Data Fusion for INS/GPS/SAR Integrated Navigation System," Aerospace Science and Technology, vol. 13, pp. 232 - 237, 2009.

[15] C. A. Balanis, Antenna theory: analysis and design. John Wiley \& Sons, 2012.

[16] S. Nassar, K.-P. Schwarz, and N. El-Sheimy, "INS and INS/GPS Accuracy Improvement Using Autoregressive (AR) Modeling of INS Sensor Errors," in Proceedings of the 2004 National Technical Meeting of The Institute of Navigation, 2001, pp. 936-944.

[17] M. Obst, S. Bauer, and G. Wanielik, "Urban Multipath Detection and Mitigation with Dynamic 3D Maps for Reliable Land Vehicle Localization," in Position Location and Navigation Symposium (PLANS), 2012 IEEE/ION. IEEE, 2012, pp. 685-691.

[18] L. Wang, P. D. Groves, and M. K. Ziebart, "GNSS Shadow Matching: Improving Urban Positioning Accuracy using a 3D City Model with Optimized Visibility Prediction Scoring," 2012.

[19] A. Lewandowski, B. Niehöfer, and C. Wietfeld, "Performance Evaluation of Satellite-based Search and RescueServices: Galileo vs. Cospas-Sarsat," in The 68th IEEE Vehicular Technology Conference (VTC). Calgary, Canada: IEEE, September 2008.

[20] B. Niehoefer, S. Subik, and C. Wietfeld, "The CNI Open Source Satellite Simulator based on OMNeT++," in OMNeT++ Workshop 2013 - 6th International Workshop to be held in conjuntion with SIMUTools, 2013.

[21] F. Torán-Martí, J. Ventura-Traveset, and J. C. de Mateo, "The ESA SISNET Project: Real-Time Access to the EGNOS Services across the Internet," in 2nd ESA ESTB Workshop, 2001.

[22] B. Hofmann-Wellenhof, H. Lichtenegger, and J. Collins, "Global positioning system. theory and practice." Global Positioning System. Theory and practice., by Hofmann-Wellenhof, B.; Lichtenegger, H.; Collins, J.. Springer, Wien (Austria), 1993, 347 p., ISBN 3-211-82477-4, ISBN 0-387-82477-4 (USA)., 2001.

[23] J. P. Collins and R. B. Langley, "Nominal and Extreme Error Performance of the UNB3 Tropospheric Delay Model," Geodesy and Geomatics Engineering Technical Reports; 204, 1999.

[24] G. Girau, A. Tomatis, F. Dovis, and P. Mulassano, "Efficient Software Defined Radio Implementations of GNSS Receivers," in Circuits and Systems, 2007. ISCAS 2007. IEEE International Symposium on, 2007, pp. $1733-1736$.

[25] B. Niehoefer, F. Schweikowski, and C. Wietfeld, "Smart Constellation Selection for Precise Vehicle Positioning in Urban Canyons using a Software-Defined Receiver Solution," in 20th Symposium on Communications and Vehicular Technology, Namur, Belgium, Nov. 2013.

\section{BIOGRAPHY}

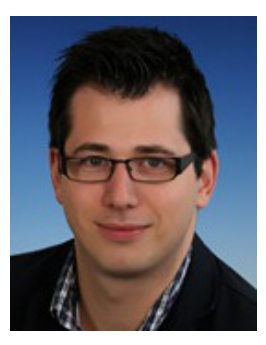

Brian Niehoefer obtained his diploma in Electrical Engineering from the $T U$ Dortmund University in Germany in 2008. He is currently employed as a researcher at the Communication Networks Institute at the same university. His research interests include the conceptual design and evaluation of multipath compensation techniques for global navigation satellite systems and the development of a Multiscale Simulation Environment for the performance evaluations of satellite systems and led to 15 publications and two Best Paper Awards so far.

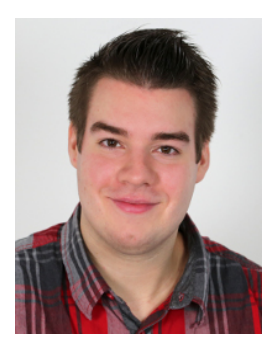

Florian Schweikowski achieved his master of science in Electrical Engineering at the TU Dortmund University in Germany. He is currently a researcher at the Communication Networks Institute at the same university. His research interests cover the object localization in wireless near-field communication areas and object positioning especially via the GPS system.

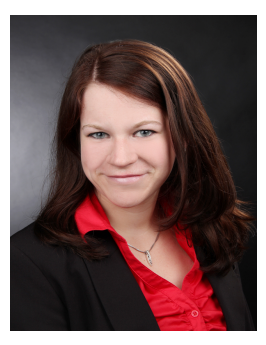

Sarah Lehnhausen received her B.Sc. (2011) and her M.Sc.(2013) in Electrical Engineering at the TU Dortmund University in Germany. Since 2009 she was employed as student assistant at the Communication Network Institute. From February 2014 she is working at ESTEC(ESA) in the GNSS Evolution Programme as a trainee with a scholarship from the German Aerospace Center.

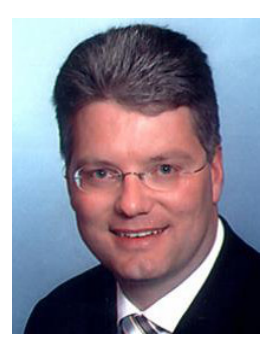

Prof. Dr.-Ing. Christian Wietfeld received his Dr.-Ing. degree from RWTH Aachen University. He is now the head of the Communication Networks Institute (CNI) of TU Dortmund University, Germany. For 20 years he has initiated and contributed to national and international research and development projects on wireless data communication systems in academia and industry. He has published over 145 peer-reviewed publications and holds several patents. For his contributions to the standardization of next generation mobile network architectures, he received in 1999 an ITU-T outstanding contribution award. His current research interests include the system design, modeling and performance evaluation of communication networks in challenging environments. Christian Wietfeld is a Senior Member of IEEE and chairs the IEEE's German sister organization VDE/ITG committee on "Communication Networks and Systems". He has co-founded the IEEE GLOBECOM Workshop on Wireless Networking for Unmanned Autonomous Vehicles (Wi-UAV). 